\section{GPR91 goes local}

\section{By Lev Osherovich, Senior Writer}

GPR91 is a key player in autoimmune disease, transplant rejection and diabetic retinopathy, according to new findings emerging from both pharmaceutical and academic laboratories. Taken together, the research suggests targeting the $G$ protein-coupled receptor may help prevent local inflammatory and angiogenic effects caused by succinate released by damaged tissue.

Succinate is a short-lived intermediate of the Krebs cycle, an energy-generating process that occurs inside mitochondria. The new papers suggest that succinate receptor 1 (GPR91) is a first responder to succinate released by damaged mitochondria and dying cells, triggering inflammation and vascular proliferation (see Figure 1, "Sensing succinate").

Novartis AG, whose scientists reported on GPR91's role in autoimmune disease and transplant rejection in Nature Immunology, ${ }^{1}$ is moving ahead with a preclinical GPR91 program for autoimmune disease. Meanwhile, a University of Montreal group that published a Nature Medicine paper focused on diabetic retinopathy ${ }^{2}$ is developing GPR91 modulators for retinopathies.

These indications could breathe new life into GPR91, which researchers at Tularik Inc. suggested as a target for renal complications of diabetes in 2004. ${ }^{3}$ A spokesperson for Amgen Inc., which acquired Tularik later that year, told SciBX that the company is no longer pursuing GPR91 research.

Also, Pfizer Inc. shelved its GPR91 program for diabetic nephropathy after finding that the high levels of circulating succinate found in mouse and rat models of hypertension and diabetes were not duplicated in human hypertensives and diabetics.

\section{Innate succinate}

Researchers at the Novartis Institutes for Biomedical Research (NIBR) reported in their Nature Immunology paper that GPR91 helps activate dendritic cells (DCs). DCs act as sentinels to detect pathogens and injured tissue, but they can promote inflammation and autoimmunity when stimulated inappropriately.

José Carballido, executive director of autoimmunity, inflammation and transplantation at NIBR and senior author of the paper, told SciBX that his team learned prior to 2004 that GPR91 was expressed in DCs. However, it was the Tularik group's identification of succinate as the likely natural ligand for GPR91 that allowed Carballido's team to figure out the receptor's role in immune activation.

Carballido's group found that immature human DCs treated with succinate showed higher intracellular calcium levels, more mitogen- activated protein kinase activity, higher cytokine secretion and greater migration-all signs of DC activation-than untreated controls. The team obtained similar results with mouse DCs and found that DCs from GPR91 knockout mice did not respond to succinate.

Although only a modest DC activator by itself, succinate increased the potency of toll-like receptor (TLR) ligands. TLRs are thought to be the primary sensors of immunological distress, and they help DCs detect cellular debris and pathogen-associated molecules.

In mouse DCs, succinate boosted cytokine production elicited by TLR3 and TLR7 agonists compared with that seen in untreated controls. In contrast, GPR91 knockout DCs did not show enhanced TLR activation after succinate treatment.

GRP91 knockout mice also had lower levels of T cell activation in response to tetanus toxin than wild-type controls. In addition, GPR91deficient skin grafts survived longer than wild-type grafts when transplanted onto immunologically incompatible mice.

Collectively, the results show that the innate immune system is not as sensitive to pathogens or cellular damage when it can't sense succinate. "We defined a mechanism that could activate the innate immune system," said Carballido.

Novartis is playing both sides of the coin-antagonizing GPR91 to lower DC activation in autoimmune disease and inflammation, and agonizing it to boost vaccine efficacy.

"Based on our studies, GPR91 is a good target for autoimmune diseases," said Carballido. "Interfering with GPR91 signaling could reduce the effect of presentation of antigens that trigger autoimmune responses and graft rejection."

On the flip side, he noted that agonists of GPR91 could potentially break tolerance and hence be used to design new vaccine adjuvants.

Carballido told SciBX that GPR91 is now a focus of the company's autoimmune disease drug discovery efforts. Novartis has patented the technology published in Nature Immunology and is developing small molecule modulators of GPR91, according to Carballido.

He added that "Novartis is open to cooperation" with other parties aiming to facilitate the development of GPR91-based therapeutics.

\section{Eye eye}

In the Nature Medicine paper, the University of Montreal team found that succinate sensing by GPR91 triggers angiogenesis in the retina in response to hypoxia-induced cell damage.

Abnormal retinal angiogenesis is a hallmark of diseases such as diabetic retinopathy and wet age-related macular degeneration (AMD). The article pinpoints the retinal ganglion cells (RGCs) behind the retina's photoreceptors as the source of proangiogenic factors, and it points to GPR91 as an attractive target upstream of VEGF.

VEGF stimulates excessive vascularization in the damaged retina and is the target of the market-leading AMD drug, Lucentis ranibizumab from Genentech Inc. and Novartis. The drug also is in Phase III trials for diabetic macular edema (DME), which precedes fullblown retinopathy.

Although multiple companies are targeting VEGF and other proangio- 


\section{TARGETS \& MECHANISMS}

genic factors in the retina, ${ }^{4}$ the Nature Medicine paper represents "the first time that we know where the angiogenic factors come from," said Sylvain Chemtob, professor of pediatrics and pharmacology at the University of Montreal and senior author on the paper.

Chemtob's group showed that succinate levels spiked in ischemic rat retinas compared with those seen in untreated controls. Ischemia, or lack of blood flow, promotes growth of vascular tissue. Indeed, the team found that injecting succinate into the eye promoted vascular outgrowth even without ischemic conditions. Succinate stimulated the production of proangiogenic factors such as VEGF and angiopoietin 1 and angiopoietin 2.

Small interfering RNA knockdown of GPR91 in fetal rats blocked the proangiogenic effects of succinate, unlike control siRNA targeting other genes. Likewise, knocking down GPR91 blocked the production of angiogenic factors in succinate-treated retinas compared with what was seen in mock-treated controls.

When Chemtob's team probed the rat retina with a GPR91specific antibody, they found that the succinate receptor was abundantly expressed in the RGCs.

To prove that RGCs sense succinate and promote angiogenesis, the researchers briefly stimulated cultured rat RGCs with succinate and then collected the surrounding cell culture medium. When pipetted onto aortic explants, this conditioned medium elicited angiogenesis, unlike medium from unstimulated controls.

In a similar experiment with RGCs subjected to GPR91 knockdown, there was much less angiogenesis than that seen in untreated controls.

Thus, Chemtob's team reasoned that blocking GPR91 could pro-

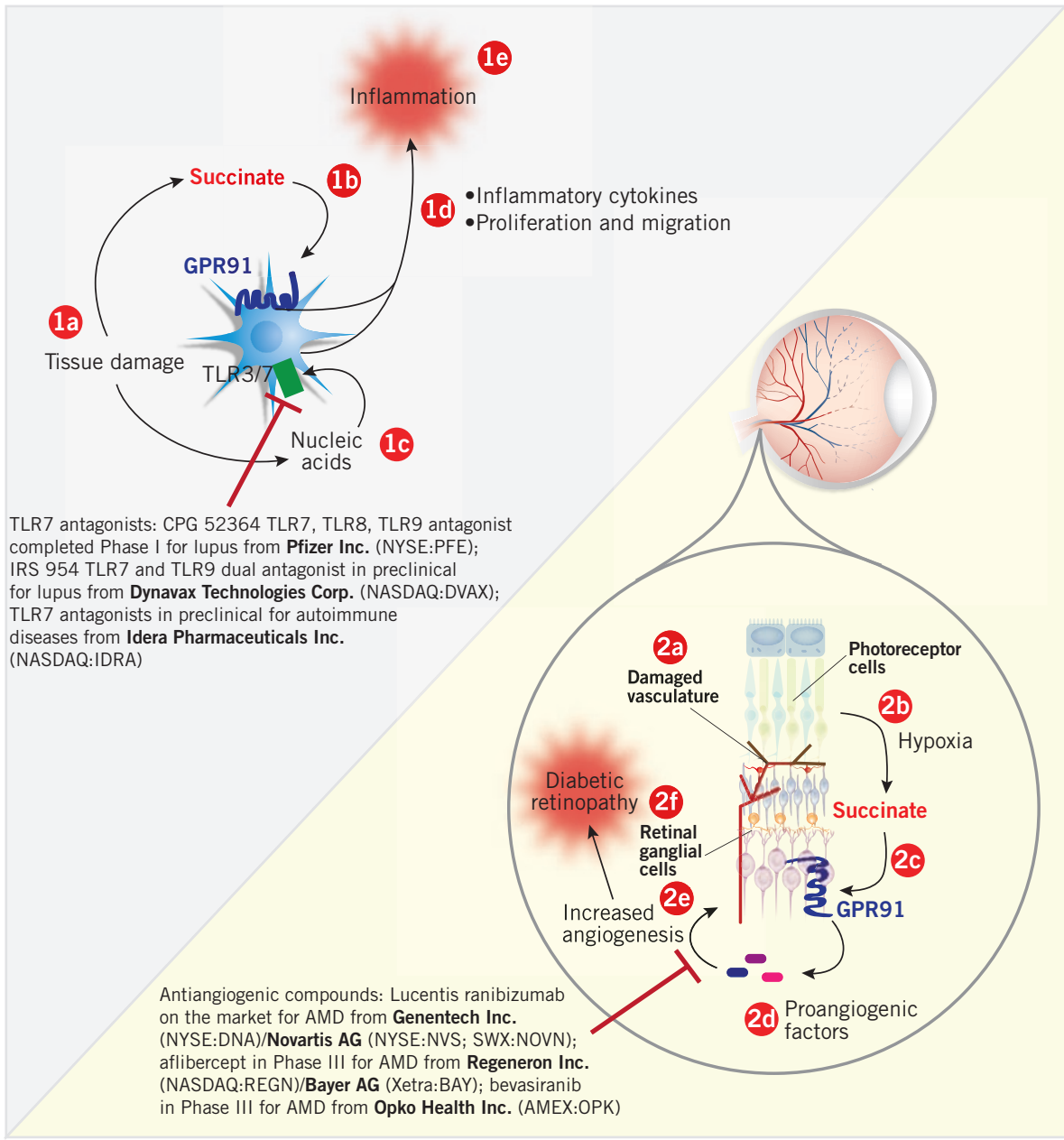

Figure 1. Sensing succinate. Compounds targeting succinate receptor 1 (GPR91) could be useful for autoimmune- and eye-related conditions, including diabetic retinopathy and graft rejection.

In dendritic cells (DCs), succinate is released as a result of nearby tissue damage [1a]. Rubic et al. report that in DCs, succinate sensing by GPR91 [1b] synergizes with sensing of nucleic acids by toll-like receptor 3 (TLR3) and TLR7 [1c] to enhance innate immune cell activation and production of inflammatory cytokines [1d]. DC activity can lead to inflammation, autoimmunity or transplant rejection [1e].

In the retina of diabetics, damage to vascular tissue [2a] leads to hypoxia [2b] and triggers the release of succinate, a metabolite that acts as an alarm signal. Sapieha et al. show that neurons in the back of the retina detect succinate through GPR91 [2c] and respond by secreting proangiogenic factors including VEGF and angiopoietins [2d], which stimulate angiogenesis [2e]. Excessive vascularization caused by this cycle leads to diabetic retinopathy and vision loss [2f]. 


\section{TARGETS \& MECHANISMS}

tect against proliferative retinopathies such as those caused by DME. Indeed, the team knocked down GPR91 in rats with experimentally induced retinal ischemia and observed lower levels of vascularization and retinopathy than those seen in mock-treated controls.

Altogether, the Nature Medicine article makes a case for targeting GPR91 in the RGCs using the same kind of selective antagonists that Novartis is developing for autoimmune diseases.

"I'm really excited about the retina study because of the association between kidney and retinal disease in diabetes," said Janos PetiPeterdi, associate professor of physiology, biophysics and medicine at the University of Southern California. "Ultimately, it's all connected to blood supply."

In June, Peti-Peterdi and co-workers reported a role for succinate and GPR91 in diabetic nephropathy. ${ }^{5}$ In the kidney, GPR91 stimulates the renin-angiotensin system, a hormone cascade that leads to hypertension and edema. ${ }^{6}$ Peti-Peterdi noted that the reninangiotensin system also works in the retina to promote edema, a hallmark of diabetic retinopathy.

Thaddeus Dryja, head of ophthalmology translational medicine at NIBR, told SciBX that Chemtob's paper provides a hint as to why DME affects the retina but not other parts of the nervous system. The Montreal study showed that GPR91 was not present in neurons adjacent to the RGC layer.

Paul Olson, CSO of Potentia Pharmaceuticals Inc., believes the two studies provide a mechanism for the recently discovered role for innate immunity in proliferative retinopathy.

Together, he said, the studies "have identified the stimulating compound, the receptor it signals and the cell type that responds" to hypoxia-induced activation of innate immunity in the retina.

Potentia is developing inhibitors of the complement system to treat retinal angiopathies. Compstatin (POT-4), a synthetic peptide inhibitor of complement component 3, is in Phase I for dry AMD.

A spate of recent studies has implicated the complement cascade, one arm of the innate immune system, in various forms of hereditary retinopathy and AMD.? Migratory immune cells such as macrophages are thought to deposit complement factors in the retina in response to cellular damage.

Chemtob, however, doesn't believe that innate immune cells are behind retinopathy. He noted that his team's experiments with cultured RGCs appear to rule out a role for other immune cells. It thus remains to be seen whether GPR91 in RGCs affects complement production in the retina.

The Montreal team is now developing compounds that modulate GPR91, as well as investigating sustained-release methods for delivering such compounds to the retina.

The group's findings have not been patented. Chemtob said his team is willing to collaborate with companies interested in targeting GPR91.

\section{REFERENCES}

1. Rubic, T. et al. Nat. Immunol.; published online Sept. 28, 2008; doi:10.1038/ni.1657

Contact: José M. Carballido, Novartis Institutes for Biomedical Research, Vienna, Austria e-mail: jose.carballido@novartis.com

2. Sapieha, P. et al. Nat. Med.; published online Oct. 5, 2008; doi:10.1038/nm.1873

Contact: Sylvain Chemtob, University of Montreal, Montreal, Quebec, Canada

e-mail: sylvain.chemtob@umontreal.ca

3. He, W. et al. Nature 429, 188-193 (2004)

4. Bernstein, K. \& McCallister, E. BioCentury 16(43), A1-A10; Sept. 29, 2008

5. Toma, I. et al. J. Clin. Invest. 118, 2526-2534 (2008)

6. Fulmer, T. SciBX 1(22), 5-7; June 26, 2008

7. Rattner, A. \& Nathans, J. Nat. Rev. Neurosci. 7, 860-872 (2006)

COMPANIES AND INSTITUTIONS MENTIONED

Amgen Inc. (NASDAQ:AMGN), Thousand Oaks, Calif. Genentech Inc. (NYSE:DNA), South San Francisco, Calif. Novartis AG (NYSE:NVS; SWX:NOVN), Basel, Switzerland Pfizer Inc. (NYSE:PFE), New York, N.Y. Potentia Pharmaceuticals Inc., Louisville, Ky. University of Montreal, Montreal, Quebec, Canada University of Southern California, Los Angeles, Calif. 